\title{
Corrigendum
}

\section{ATP11B mediates platinum resistance in ovarian cancer}

Myrthala Moreno-Smith, J.B. Halder, Paul S. Meltzer, Tamas A. Gonda, Lingegowda S. Mangala, Rajesha Rupaimoole, Chunhua Lu, Archana S. Nagaraja, Kshipra M. Gharpure, Yu Kang, Cristian Rodriguez-Aguayo, Pablo E. Vivas-Mejia, Behrouz Zand, Rosemarie Schmandt, Hua Wang, Robert R. Langley, Nicholas B. Jennings, Cristina Ivan, Jeremy E. Coffin, Guillermo N. Armaiz, Justin Bottsford-Miller, Sang Bae Kim, Margaret S. Halleck, Mary J.C. Hendrix, William Bornman, Menashe Bar-Eli, Ju-Seog Lee, Zahid H. Siddik, Gabriel Lopez-Berestein, and Anil K. Sood

Original citation: J Clin Invest. 2013;123(5):2119-2130. https://doi.org/10.1172/JCI65425.

Citation for this corrigendum: J Clin Invest. 2021;131(7):e149893. https://doi.org/10.1172/JCI149893.

The Editors previously posted an Expression of Concern for this article regarding images in Figure 3B that appeared to be the same as images that were previously published in a 2009 Clinical Cancer Research paper with different sample labels (doi: $10.1158 / 1078-0432$. CCR-08-2306). Additionally, the empty liposome and control siRNA PCNA-stained panels were noted to appear to be the same. An institutional review committee concluded that the errors in Figure 3B were inadvertent and recommended correction. The authors provided the correct version of Figure 3B with data obtained from a repeated experiment. That correct version is shown below.

The authors regret the errors.
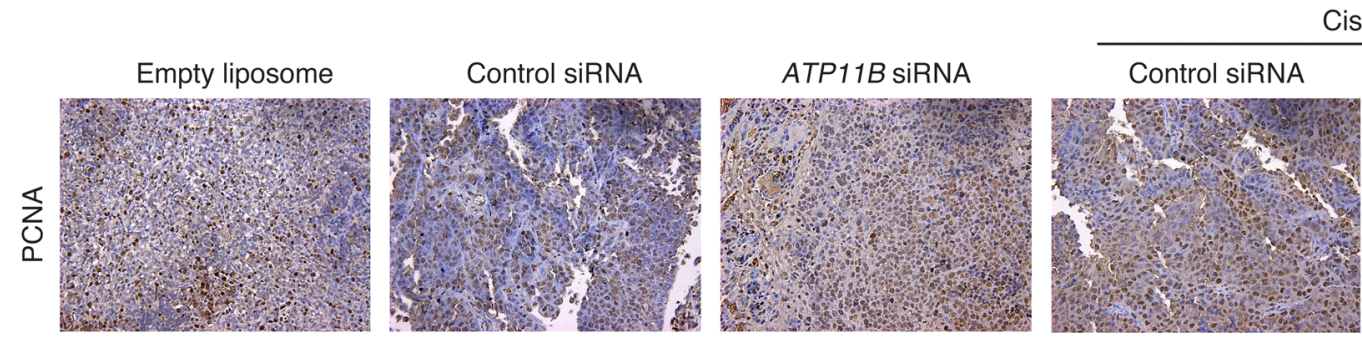

Cisplatin
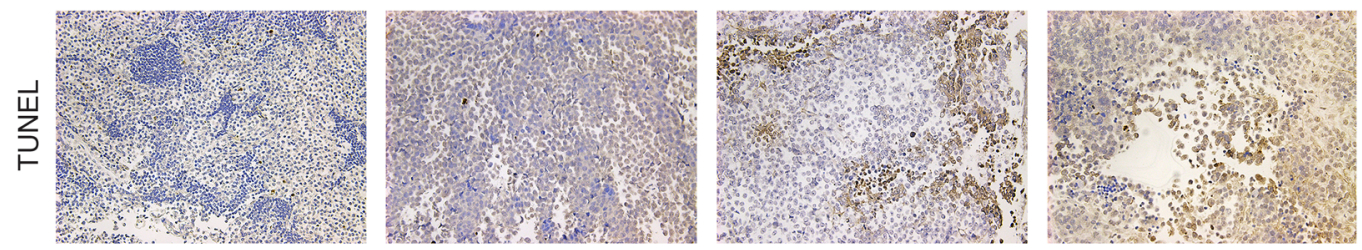

ATP11B SiRNA
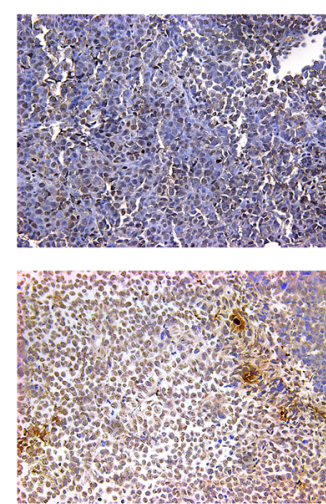